\title{
Development of an acoustic sensor for the future IceCube-Gen2 detector for neutrino detection and position calibration
}

\author{
Stefan Wickmann ${ }^{1, \star}$, Dmitry Eliseev ${ }^{1}$, Dirk Heinen ${ }^{1}$, Peter Linder ${ }^{1}$, Martin Rongen ${ }^{1}$, Franziska \\ Scholz ${ }^{1}$, Lars Steffen Weinstock ${ }^{1}$, Christopher Wiebusch ${ }^{1}$, and Simon Zierke ${ }^{1}$ \\ ${ }^{1}$ III. Physikalisches Institut B, RWTH Aachen University, D-52056 Aachen, Germany
}

\begin{abstract}
For the planned high-energy extension of the IceCube Neutrino Observatory in the glacial ice at the South Pole the spacing of detector modules will be increased with respect to IceCube. Because of these larger distances the quality of the geometry calibration based on pulsed light sources is expected to deteriorate. To counter this an independent acoustic geometry calibration system based on trilateration is introduced. Such an acoustic positioning system (APS) has already been developed for the Enceladus Explorer Project (EnEx), initiated by the DLR Space Administration. In order to integrate such APS-sensors into the IceCube detector the power consumption needs to be minimized. In addition, the frequency response of the front end electronics is optimized for positioning as well as the acoustic detection of neutrinos. The new design of the acoustic sensor and results of test measurements with an IceCube detector module will be presented.
\end{abstract}

\section{Introduction}

The IceCube Neutrino Observatory [1], located at the geographical South Pole, instruments about one cubic kilometer of Antarctic ice to detect neutrinos. The detector consists of 86 strings, each with 60 digital optical modules (DOM) forming a photomultiplier (PMT) array with a spacing of $125 \mathrm{~m}$ between the strings. Most of the DOMs are equally spaced $(17 \mathrm{~m})$ among the strings at a depth between $1450 \mathrm{~m}$ and $2450 \mathrm{~m}$ beneath the ice surface. Geometry calibration of the detector is performed with deployment data of the ice drilling and an optical calibration system, with which an accuracy of less than $1 \mathrm{~m}$ can be achieved.

IceCube-Gen2 is a planned extension of IceCube [2]. It will allow for the detection of highenergetic neutrinos with an increased detection rate and precision. To accomplish this an extension of the detector volume is planned by adding more strings to the existing IceCube array. The spacing between these additional strings will therefore be increased to $240 \mathrm{~m}-300 \mathrm{~m}$, depending on the planned configurations of IceCube-Gen2.

Because of the larger spacing between the strings the quality of the optical geometry calibration is expected to deteriorate due to the extinction length $(20 \mathrm{~m}-200 \mathrm{~m})$ [3] of optical signals in the Antarctic ice. A possible solution could be the use of an independent geometry calibration system based on acoustic signals due to their larger attenuation length ( 300 $\mathrm{m},[4])$.

^e-mail: wickmann@physik.rwth-aachen.de 
The idea is to integrate acoustic sensors into the IceCube-Gen2 DOMs to use them for acoustic positioning independent of optical properties. In addition, such sensors could also be used for acoustic neutrino detection based on the thermo-acoustic effect [5] [4].

The required steps to integrate such a system into IceCube-Gen2 would be to test the concept of acoustic positioning with a DOM, to develop a suitable acoustic sensor for the required frequency ranges of the acoustic positioning and the acoustic neutrino detection with the desired low power consumption and finally to integrate the sensor into the IceCube-Gen2 DOMs. Acoustic emitters could be deployed as separate modules and do not need to be integrated into the DOMs.

Such a sensor for in-ice acoustic positioning has already been developed by the Enceladus Explorer Project (EnEx) [6]. Within this project navigation technology for a future space mission has been developed. A main aspect of the project has been the development of an in-ice acoustic positioning system for a maneuverable melting probe. The system measures the propagation time of acoustic signals between six ultrasonic emitters at the ice surface and four acoustic sensors located in the melting head of the probe. The probes position is then determined by trilateration with an accuracy of better than $1 \mathrm{~m}$ which is comparable to the accuracy required for IceCube's geometry calibration with optical signals.

The acoustic sensor developed in the EnEx-project is a piezo-based sensor, optimized for ultrasonic signals $(18 \mathrm{kHz})$ [7]. It consists of a piezo disc $(\varnothing 16 \mathrm{~mm} \times 3 \mathrm{~mm})$ and two stacked sensor front end boards. One board amplifies, filters and digitizes the received signals and the second board converts the single-ended digital signals to LVDS signals. The two front end boards and the piezo disc are encapsulated in a brass housing $(\varnothing 20 \mathrm{~mm})$.

The sensor front end contains two channels. After passing different filters the signal is digitized for each channel with a sampling rate of up to $3 \mathrm{MS} / \mathrm{s}$ at a resolution of 12 bits. The power consumption of one sensor front end with two channels is about $150 \mathrm{~mW}(30 \mathrm{~mA}$ at $5 \mathrm{~V})$.

\section{Prototype Development}

To meet the requirements of IceCube-Gen2 the EnEx acoustic sensor front end was modified in respect of the desired frequency ranges and power consumption [8]. The new prototype front end utilizes two channels as well: channel one with a frequency range from $16 \mathrm{kHz}$ to $20 \mathrm{kHz}$, optimized with regard to noise suppression of the acoustic positioning and channel two with a frequency range from $10 \mathrm{kHz}$ to $100 \mathrm{kHz}$, optimized with regard to sensitivity of the acoustic neutrino detection. To substantially reduce the power consumption the LVDS conversion was removed which also leads to a reduced pin count of the sensor front end.

The power consumption of the new prototype front end was reduced to typically $50 \mathrm{~mW}$ (max. $53 \mathrm{~mW}$ ) in idle mode (no digitization), which is about $33 \%$ compared to the original EnEx front end, and typically $75 \mathrm{~mW}$ (max. $78 \mathrm{~mW}$ ) when digitizing both channels. Further improvement could be achieved by implementing a one channel operating mode, where only one channel is digitized and the other is in idle mode which will lead to an estimated power consumption of about $35 \mathrm{~mW}$. By using enhanced electronic components optimized for low power application further reduction is possible.

A basic FPGA-firmware module was developed which controls and reads-out the prototype board. The Firmware was designed as a QSys component for use with the Altera QSys build system which will likely be used in IceCube-Gen2.

\section{Test Measurements}

The acoustic positioning concept for IceCube-Gen2 and its performance was evaluated in water (swimming pool) [8]. For this purpose, three EnEx acoustic sensors were integrated into an Ice- 
Cube DOM ${ }^{1}$ (Acoustic DOM) along with a data acquisition system (see fig. 1). The sensors were mounted into the Acoustic DOM using an aluminum construction that was glued to the glass sphere with vacuum cups. The sensor itself was pressed towards the glass sphere with metal springs (see fig. 1).

The Acoustic DOM was mounted under a floating platform and held under water with weights. On top of the floating platform two targets were mounted for reference measurements with a laser range-finder (LRF): one to measure the position of the Acoustic DOM and another to determine its heading. In addition six EnEx acoustic emitters were distributed in the swimming pool at different depths to reconstruct the position of the Acoustic DOM via acoustic measurements.
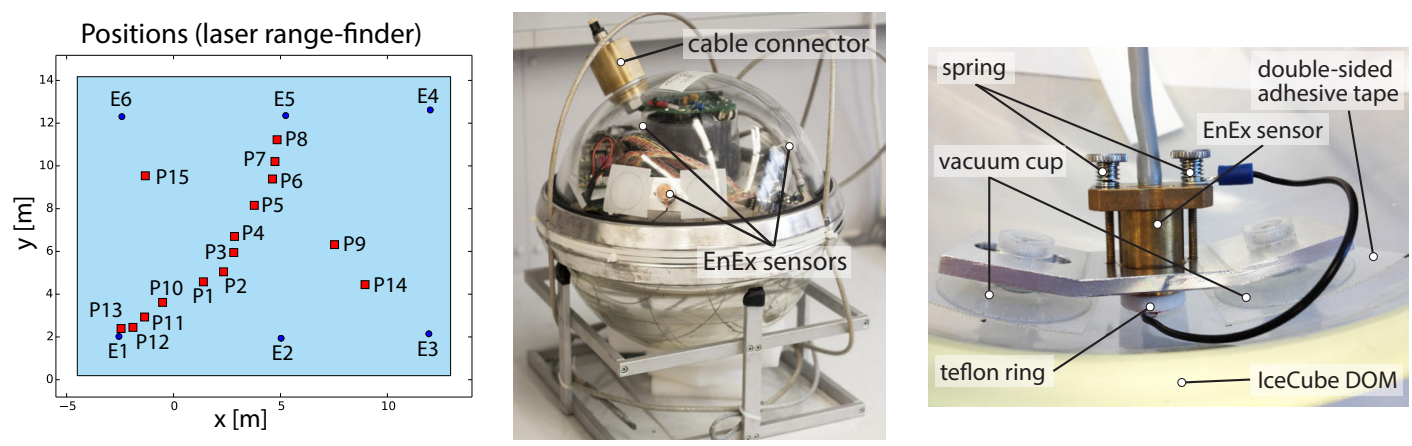

Figure 1. left: positions of the six acoustic emitters (E1 - E6, circles) and 15 measurement positions of the Acoustic DOM (P1 - P15, rectangles) in the swimming pool, measured with the laser range-finder (LRF); center: Acoustic DOM: IceCube DOM ${ }^{1}$ with three EnEx sensors, read-out electronics and a pressure-tight cable connector; right: mounting of the acoustic sensor inside the IceCube DOM.

After measuring the positions of the six acoustic emitters with the LRF, the measurement procedure continues as follows: First, the reference position and heading of the Acoustic DOM at the respective measurement position is measured with the LRF. Then a sine-burst signal is sent consecutively from each emitter and data is taken synchronously with the Acoustic DOM for each emitter. After changing the position of the Acoustic DOM the above steps are repeated (see fig. 1). The position was reconstructed by determining the propagation time between the acoustic emitters and the acoustic sensors by detecting the start of the sine-burst as the point, where the signal exceeds $5 \sigma$ of the preceding noise signal. After that, a least square fit was applied to the measured data for each channel of the sensors to reconstruct the position of the sensor.

\section{Performance}

The reconstructed positions are in good agreement with the reference positions measured with the LRF and the heading of the Acoustic DOM can be estimated (see fig. 2). However, there are some systematic errors that are not yet fully understood.

To quantify the performance of the system, the distance of the measured position from the reference position (fig. 2), both in full space and in the $x-y-p l a n e$, were histogrammed. The accuracy in the $\mathrm{x}-\mathrm{y}$-plane (median: $6.31 \mathrm{~cm}$ ) is better than the overall 3D accuracy (median: $26.48 \mathrm{~cm}$ ) which results from smaller variation of the acoustic emitters' positions in z-direction compared to the $x$-y-plane, due

\footnotetext{
${ }^{1}$ kindly provided by Rolf Nahnhauer, DESY
} 

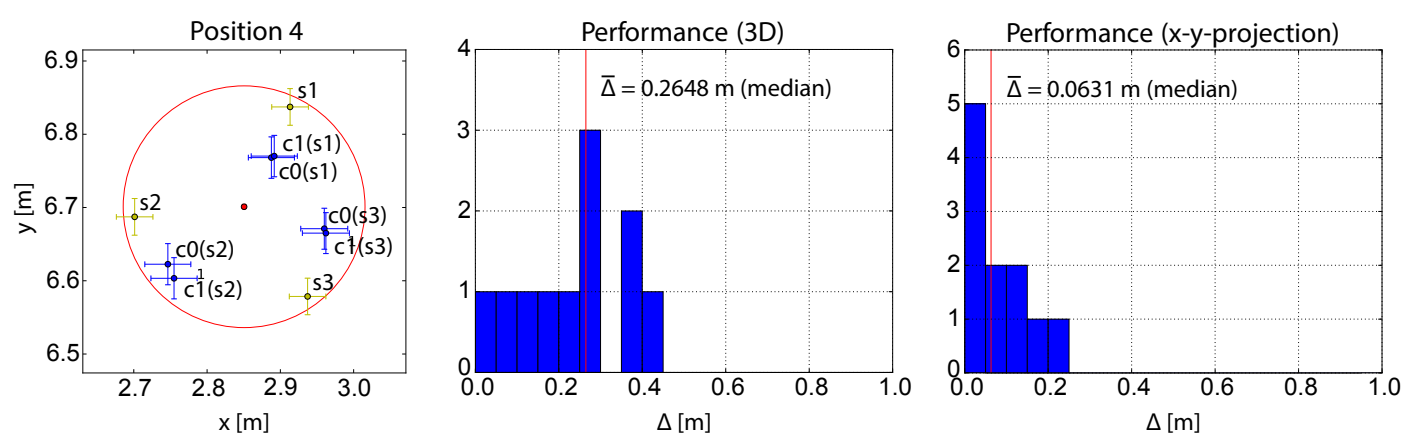

Figure 2. left: reconstructed positions of the acoustic sensors at Acoustic DOM pos. 4, cX(Y) labels channel $\mathrm{X}$ of sensor $\mathrm{Y}, \mathrm{s} 1$ - $\mathrm{s} 3$ are the reference positions of the acoustic sensors deduced from the measurements with the LRF, the circle shows the diameter of the Acoustic DOM; center, right: Histogram of the distances of the acoustically measured positions from the true positions in full space and in the $\mathrm{x}$-y-plane, respectively.

to the limited depth of the swimming pool. It is expected that a more sophisticated data selection for noise and outliers, as well as orientation dependent corrections would further improve the accuracy.

For use in ice, the media properties involve larger uncertainties which, however, do not vary with time. A similar performance is expected if the system provides sufficient redundancy for determining and correcting these uncertainties.

\section{Conclusion}

A concept of an acoustic positioning system for IceCube-Gen2 based on the EnEx positioning system has been evolved by developing a prototype sensor front end based on the EnEx sensor front end and a firmware module for IceCube-Gen2. The frequency ranges of the front end have been adapted to meet the requirements of IceCube-Gen2. Also the power consumption of the front end was reduced to a third of the power consumption of the EnEx sensor front end. The EnEx acoustic sensor has been integrated into an IceCube DOM and the acoustic positioning of an IceCube detector module has been demonstrated in water with a measured accuracy better than $7 \mathrm{~cm}$ on average.

The next steps to further evolving the concept of acoustic positioning for IceCube-Gen 2 would be to improve the prototype front end by means of power consumption and actually integrate it into an IceCube-Gen2 DOM. Furthermore, a concept for design and distribution of acoustic emitters for IceCube-Gen2 needs to be developed.

\section{References}

[1] A. Achterberg et al., Astroparticle Physics 26, 155 (2006)

[2] M.G. Aartsen et al. (2014), arXiv: 1412.5106

[3] M. Aartsen et al., Nuclear Instruments and Methods in Physics Research Section A 711, 73 (2013)

[4] R. Abbasi et al., Astroparticle Physics 34, 382 (2011)

[5] G. Askariyan et al., Nuclear Instruments and Methods 164, 267 (1979)

[6] J. Kowalski et al., Cold Regions Science and Technology 123, 53 (2016)

[7] D. Eliseev et al., Annals of Glaciology 55, 253 (2014)

[8] S. Wickmann, master's thesis, RWTH Aachen University (2016) 